\title{
1 KAT7 is a therapeutic vulnerability of MLL-rearranged acute myeloid leukemia
}

2

3 Yan Zi Au ${ }^{1,2}$, Muxin $\mathrm{Gu}^{2}$, Etienne De Braekeleer ${ }^{2}$, Jason $\mathrm{Yu}^{1}$, Swee Hoe Ong ${ }^{1}$, Malgorzata Gozdecka ${ }^{2,4}$,

$4 \mathrm{Xi} \mathrm{Chen}^{3}$, Konstantinos Tzelepis ${ }^{2,5}$, Brian J.P Huntly ${ }^{4,6,7}$, George Vassiliou ${ }^{2,4,6^{*}}$, Kosuke Yusa ${ }^{1,8^{*}}$

6 1. Stem Cell Genetics, Wellcome Sanger Institute, Hinxton, Cambridge, UK

7 2. Haematological Cancer Genetics, Wellcome Sanger Institute, Hinxton, Cambridge, United $8 \quad$ Kingdom

9 3. Gene Expression Genomics, Wellcome Sanger Institute, Hinxton, Cambridge, United Kingdom

10 4. Wellcome Trust-MRC Stem Cell Institute, Cambridge Biomedical Campus, University of 11 Cambridge, Cambridge, UK

12 5. Gurdon Institute, University of Cambridge, Tennis Court Road, Cambridge, UK

13 6. Department of Haematology, Cambridge University Hospitals NHS Trust, Cambridge, UK

14 7. Cambridge Institute for Medical Research and Wellcome Trust-Medical Research Council, Stem

15 Cell Institute and Department of Haematology, University of Cambridge, Cambridge, UK

16 8. Stem Cell Genetics, Institute for Frontier Life and Medical Sciences, Kyoto University, Kyoto, 17 Japan

18

19 Present address:

20 JY: Department of Cell Biology, The Francis Crick Institute, London, United Kingdom

$22 *$ Corresponding authors

23 Correspondence to: gsv20@sanger.ac.uk or k.yusa@infront.kyoto-u.ac.jp 


\section{Abstract}

25 Histone acetyltransferases (HATs) catalyze the transfer of an acetyl group from acetyl-CoA to lysine 26 residues of histones and play a central role in transcriptional regulation in diverse biological 27 processes. Dysregulation of HAT activity can lead to human diseases including developmental 28 disorders and cancer. Through genome-wide CRISPR-Cas9 screens, we identified several HATs of the 29 MYST family as fitness genes for acute myeloid leukaemia (AML). Here we investigate the 30 essentiality of lysine acetyltransferase KAT7 in AMLs driven by the MLL-X gene fusions. We found 31 that KAT7 loss leads to a rapid and complete loss of both H3K14ac and H4K12ac marks, in 32 association with reduced proliferation, increased apoptosis and differentiation of AML cells. 33 Acetyltransferase activity of KAT7 is essential for the proliferation of these cells. Mechanistically, our 34 data propose that acetylated histones provide a platform for the recruitment of MLL-fusion35 associated adaptor proteins such as BRD4 and AF4 to gene promoters. Upon KAT7 loss, these factors 36 together with RNA polymerase II rapidly dissociate from several MLL-fusion target genes that are 37 essential for AML cell proliferation, including MEIS1, PBX3 and SENP6. Our findings reveal that KAT7 38 is a plausible therapeutic target for this poor prognosis AML subtype. 


\section{Introduction}

Acute myeloid leukemia (AML) is an aggressive malignancy of haematopoietic stem and progenitor cells. Translocations involving the mixed lineage leukemia gene (MLL or KMT2A) characterize an aggressive subtype of the disease and are associated with a poor prognosis ${ }^{1-3}$. Currently, chemotherapy based on a combination of anthracyclines and purine analogues is the standard of care for AML, yet patients with leukemias driven by $M L L-X$ gene fusions commonly become refractory to such treatments ${ }^{4}$. Advances in our understanding of the molecular pathogenesis of acute leukemias driven by MLL-X fusion oncogenes have led to the development of new drugs, including inhibitors of bromodomain proteins ${ }^{5-7}$, DOT1L ${ }^{8,9}$ and MENIN ${ }^{10}$; however, none of these have demonstrated a significant clinical benefit for patients as yet. In order to identify new therapeutic approaches in AML, we and others have performed genome-wide CRISPR-Cas9 fitness screens in different subtypes of the disease including the MLL-X subtype ${ }^{11,12}$. Through our own screen data, we recently showed that GCN5 (KAT2A) and SRPK1 are novel vulnerability in AML and that sensitivity to SRPK1 inhibition is particularly associated with AML driven by MLL-X fusion oncogenes ${ }^{11,13}$. Systematic detailed analysis of our CRISPR screen dataset revealed that several members of the MYST family of histone acetyltransferases (HATs) are required for the survival of AML cell lines, with KAT7 displaying strong essentiality for AMLs driven by MLL-X oncogenes.

HATs are classified based on structural and sequence homology into distinct groups including the p300/CBP, MYST and GCN5 families ${ }^{14}$. They play a central role in transcriptional regulation through their function to catalyze the transfer of acetyl groups from acetyl-CoA to the $\varepsilon$-amino group of lysine residues of histones. Dysregulation of HATs is known to be associated with human diseases including developmental disorders and cancer ${ }^{14-16}$. The MYST family of HATs, characterized by their conserved MYST catalytic domain, includes KAT5 (TIP60), KAT6A (MOZ and MYST3), KAT6B (MORF and MYST4), KAT7 (HBO1 and MYST2) and KAT8 (MOF) ${ }^{15,16}$. While KAT6A and KAT6B are known targets of chromosomal translocations that drive $A \mathrm{ML}^{17-21}$ and KAT8 can play a role in some cases of $A M L{ }^{22}$, it is currently not known whether KAT7 plays a role in leukemogenesis. As KAT7 lacks chromatin binding domains, it relies on forming complexes with a scaffold protein (JADE or BRPF), along with EAF6 and ING4/5, for efficient histone acetylation ${ }^{23}$. The choice of the scaffold protein primarily determines target histones, namely $\mathrm{H} 3$ or $\mathrm{H} 4$. The KAT7-BRPF acetylates histone $\mathrm{H} 3$ tails $(\mathrm{K} 14 \text { and } \mathrm{K} 23)^{23-26}$, whereas KAT7-JADE complexes acetylates histone $\mathrm{H} 4$ tails (K5, K8, and K12) ${ }^{27}$, respectively. In development, abolishing KAT7 results in major reduction of H3K14ac in both mouse fetal liver erythroblasts ${ }^{24}$ and mouse embryo ${ }^{25}$; the latter associates with lethality. 
73 Here we investigate the molecular mechanism underlying the requirement of KAT7 in MLL-X-driven

74 AMLs. We report that loss of KAT7 leads to reduced proliferation and enhanced apoptosis and 75 differentiation. We also show that KAT7 is essential for maintaining the transcriptional program 76 driven by MLL-AF9, through the recruitment of BRD4 and other MLL-fusion associated proteins such 77 as AFF1 to the promoters of a subset of MLL-AF9 target genes. Together, our findings propose KAT7 78 as a plausible therapeutic target in MLL-X AML and also provide novel mechanistic insights into the 79 molecular basis of MLL-X-driven transcriptional dysregulation. 
80

81

82

83

84

85

86

87

88

89

90

91

92

93

94

95

96

97

98

99

100

101

102

103

104

105

106

107

108

109

110

111

112

113

\section{Materials and Methods}

\section{Cell culture}

MOLM-13, MV4-11, THP-1, HL-60 and K562 were cultured in RPMI 1640 medium (Thermo Fisher) supplemented with 10 \% FBS (Thermo Fisher). Nomo-1 was cultured in RPMI 1640 supplemented with $10 \%$ FBS, $1 \%$ penicillin/streptomycin (Thermo Fisher), 1 \% sodium pyruvate (Thermo Fisher), 1 \% glucose (Sigma). OCI-AML2 and OCI-AML3 were cultured in MEM- $\alpha$ (Lonza) with $20 \%$ FBS and $1 \%$ GlutaMax (Thermo Fisher). All these cell lines stably express Cas9 (ref. 11). To maintain Cas9 expression, blasticidin (Thermo Fisher) was added to MOLM-13, MV4-11, THP-1, Nomo-1, HL-60, OCI-AML2 and OCI-AML3 culture at $10 \mu \mathrm{g} / \mathrm{ml}$, and $\mathrm{K} 562$ at $15 \mu \mathrm{g} / \mathrm{ml}$. 293FT cells (Thermo Fisher) were cultured in DMEM (Thermo Fisher) supplemented with $10 \%$ FBS and $1 \%$ GlutaMax. All cell lines were incubated at $37{ }^{\circ} \mathrm{C}$ with $5 \% \mathrm{CO}_{2}$ and confirmed to be mycoplasma negative.

\section{Plasmid construction}

gRNA design and cloning: gRNAs from the genome-wide CRISPR-library used in the initial screens ${ }^{11}$ were selected for validation. Additional gRNAs were designed using the Wellcome Sanger Institute's Genome Editing (WGE) (http://www.sanger.ac.uk/htgt/wge/) ${ }^{28}$. Only gRNAs that targeted exons present in all putative transcripts and had no off-target hits with less than 3-nucleotide mismatch to their on-target sequence were selected. All gRNAs were cloned into the Bbsl site of pKLV2U6gRNA5(Bbsl)-PGKpuro2ABFP-W ${ }^{11}$. gRNAs used in this study are listed in Table S2.

gRNA-resistant wild-type and E508Q mutant KAT7 expression vector: A T2A-fused wild-type (WT) KAT7 cDNA fragment resistant to both gKAT7 (5) and gKAT7 (A10) was purchased from IDT and cloned into the BsrGI site of pKLV-EF1aGFP using a NEBuilder HiFi kit (NEB), resulting in pKLVEF1aGFP2AKAT7-W. To introduce E508Q substitution, the region between the Srfl and BsaBI sites of the KAT7 ORF was replaced with a gBlock fragment containing the substitution using NEBuilder HiFi kit, resulting in pKVL-EF1aGFP2AKAT7E508Q-W. Sequences were verified by Sanger sequencing.

KAT7 degron-AID system: The region between the BamHI and Notl of pKLV-EF1aGFPKAT7-W was replaced with a gBlock fragment appended with the degron tag using NEBuilder HiFi kit, resulting in pKLV-EF1aGFP2AKAT7-linkermAID-W. A T2A-fused Oryza sativa TIR1 (OsTIR1) fragment was purchased as a gBlock fragment and cloned into the BsrGI site of pKLV-EF1amCherry, resulting in pKVL-EF1a-mCherry-2A-OsTIR1-W. Sequences were verified by Sanger sequencing.

\section{Lentivirus production and transduction}


114 Lentivirus was produced as described previously (31). AML and CML cell lines were transduced by

115 adding lentivirus and $8 \mu \mathrm{g} / \mathrm{ml}$ polybrene (Millipore) to $3.0 \times 10^{4}$ cells per well of a 96-well plate or $1 \mathrm{x}$

$11610^{6}$ cells per well of a 6 -well plate and incubated at $37^{\circ} \mathrm{C}$ for $22 \mathrm{~h}$. Viral supernatant was then

117 replaced with fresh culture media and the cells were passaged for further culture.

\section{Cell sorting and flow cytometry analyses}

120 Cell sorting was performed using Mo-Flo XDP or BD INFLUX under containment-level 2 conditions.

121 FACS analyses were performed using BD LSRFortessa and raw files were analysed using FlowJo.

122

\section{Proliferation}

124 Cells were first transduced with gRNA-expressing lentivirus in 96-well plates as described above. The 125 percentage of BFP-positive (knock-out) cells was determined for each well every 2 days between day 1264 and day 14 post transduction using flow cytometry. Culture media were refreshed every 2 days. 127 Prior to FACS analysis, cells were fixed in $4 \%$ paraformaldehyde in phosphate-buffered saline (PBS) 128 for 10 minutes and re-suspended in $1 \%$ bovine serum albumin in PBS. Values were normalized to the BFP-positive percentage on day 4.

\section{Differentiation and apoptosis assays}

132 Cells were transduced with gRNA-expressing lentivirus in 6-well plates as described above. BFP133 positive (knock-out) cells were collected by cell sorting 3 days post transduction and further cultured until analysis. For differentiation analysis, $2.3 \times 10^{5}$ cells were harvested 7 days post transduction, washed in PBS and staining buffer (2 \% FBS in PBS), and stained with APC-conjugated CD11b (1.15 $\mu \mathrm{l}$ antibody per $100 \mu \mathrm{l}$ staining buffer per test, 17-0118, eBioscience) or APC-conjugated mouse IgG1k isotype control (17-4714, eBioscience) on ice for 30min in the dark. The cells were then washed twice with staining buffer and analysed by FACS. For apoptosis assays, $2.3 \times 10^{5}$ cells were analysed 9 days post transduction using Annexin V Apoptosis Detection Kit APC (88-8007, eBioscience) according to the manufacturer's instruction.

Generation of Auxin Inducible Degron (AID) KAT7 protein degradation system and treatment with indole-3-acetic acid (IAA)

144 MOLM-13 cells were first transduced with lentivirus produced with pKLV-

145 EF1aGFP2AKAT7_linkermAID-W and GFP-positive cells were sorted. The cells were then transduced 146 with lentivirus produced with pKLV2-U6gKAT7(A10)-PGKpuro2ABFP-W to knock out the endogenous 147 KAT7 gene and sorted for BFP-positive cells to enrich for the knock-out (KO) population. GFP-BFP- 
148

149

150

151

152

153

154

155

156

157

158

159

160

161

162

163

164

165

166

167

168

169

170

171

172

173

174

175

176

177

178

179

180

181

double positive cells were further transduced with lentivirus produced with pKLV-EF1a-mCherry-2A-

OsTIR1-W and sorted for mCherry-positive cells, resulting in the generation of MOLM-13 KAT7-AID cells. Indole-3-acetic acid (IAA; Sigma) was dissolved in water at a concentration of $500 \mathrm{mM}$. MOLM13 KAT7-AID cells were treated with $500 \mu \mathrm{M}$ IAA for $24 \mathrm{~h}$ for ChIP-qPCR experiments and for $48 \mathrm{~h}$ for differentiation assays.

\section{Western blot analysis}

Cells were harvested by centrifugation and washed twice in PBS. Cell pellets were then re-suspended in NUPAGE LDS sample buffer (ThermoFisher), NuPAGE sample reducing agent (ThermoFisher) at a concentration of $1 \times 10^{6}$ cells $/ 100 \mu \mathrm{L}$. The lysates were heated at $95^{\circ} \mathrm{C}$ for $5 \mathrm{~min}$ and vortexed at room temperature for $10 \mathrm{~min}$. Equal volumes of the lysates were loaded into each well of Bis-Tris precast polyacrylamide gels (Thermo Fisher). Antibodies used are listed in Table S3.

\section{In vivo mouse work}

MOLM-13 cells expressing Cas9 and luciferase ${ }^{11}$ were transduced with lentivirus for gRNA-mediated knock-out of KAT7 using gKAT7 (A10) or empty gRNA control. BFP-positive cells were sorted 2 days post transduction and cultured. $5 \times 10^{5}$ cells were injected via the tail vein of immunocompromised NSGW41 male mice (derived by breeding NSG and KITW41 animals to homozygosity, i.e. $\mathrm{Kit}^{W 41 / \mathrm{W} 41}, \mathrm{Prkdc}^{-/}$, and $/ 12 \mathrm{rg}^{-/-}$or $\left./ 12 \mathrm{rg}^{-/ Y}\right) 3$ days post transduction. Five mice were used in each treatment group. Bioluminescence imaging was performed by injection of $200 \mu \mathrm{L}(2 \mathrm{mg} / \mathrm{mL}) \mathrm{D}$ luciferin (BioVision) per mouse. Quantification of bioluminescence was performed using the IVIS Spectrum In Vivo Imaging System(Perkin-Elmer), with Living Image version 4.3.1 software (PerkinElmer) according to the manufacturer's instructions. Log-rank (Mantel-Cox) test was used to compare survival between mouse groups. All animal studies were carried out at the Wellcome Sanger Institute under UK Home Office License PBF095404.

\section{ChIP-seq and ChIP-qPCR}

Cells were crosslinked with $1 \%$ Formaldehyde (ThermoFisher) at room temperature for 5 min (histones ChIP) or $10 \mathrm{~min}$ (non-histone ChIP), and quenched with $0.125 \mathrm{M}$ Glycine (Sigma) at room temperature for $5 \mathrm{~min}$. Cross-linked cells were washed twice with ice-cold PBS before sequential lysis with LB1 (50 mM Hepes, 140 mM NaCl, 1 mM EDTA, 10 \% glycerol, 0.5 \% NP-40, 0.25 \% Triton X100), LB2 (10 mM Tris-HCl, 200 mM NaCl, 1 mM EDTA, 0.5 mM EGTA) and LB3 (10 mM Tris-HCl, 100 $\mathrm{mM} \mathrm{NaCl}, 1 \mathrm{mM}$ EDTA, 0.5 mM EGTA, 0.1 \% Na-Deoxycholate, 0.5 \% N-lauroylsarcosine) on ice for 10 min each. Lysed samples were then sonicated for 10 and 11 cycles for MOLM-13 and OCI-AML3 
182 respectively, using the Bioruptor Pico instrument (Diagenode). Triton- $X$ was added to the sonicated

183 samples to a final concentration of $1 \%$ before centrifugation at $20,000 \times \mathrm{g}$ at $4{ }^{\circ} \mathrm{C}$ for $10 \mathrm{~min} .10 \%$

184 (by volume) of each sample was kept as input. Lysates were incubated with antibody (Table S2) at 4

$185{ }^{\circ} \mathrm{C}$ overnight with rotation. Dynabeads Protein A/G (ThermoFisher) were added the next day and 186 incubated at $4{ }^{\circ} \mathrm{C}$ for $4 \mathrm{~h}$ with rotation. ChIP samples were washed with RIPA wash buffer (50 mM 187 Hepes, 500 mM LiCl, 1 mM EDTA, 1 \% NP-40, 0.7 \% Na-Deoxycholate) 3 times, followed by wash with 188 annealing buffer (TE, $50 \mathrm{mM} \mathrm{NaCl}$ ). Elution buffer (1 \% SDS, $50 \mathrm{mM}$ Tris-HCl, $10 \mathrm{mM}$ EDTA) was 189 added to each ChIP sample and the samples were then heated at $65{ }^{\circ} \mathrm{C}$ for $30 \mathrm{~min}$. Beads were 190 subsequently removed and samples heated at $65{ }^{\circ} \mathrm{C}$ overnight to reverse cross-linking whilst 191 supplemented with $0.2 \mathrm{mg} / \mathrm{mL}$ RNase A (ThermoFisher). $0.2 \mathrm{mg} / \mathrm{mL}$ Proteinase $\mathrm{K}$ (Life Technologies) 192 was added the next day to digest proteins by incubating and shaking at $450 \mathrm{rpm}$ at $65{ }^{\circ} \mathrm{C}$ for $4 \mathrm{~h}$. 193 ChIP-DNA was purified by PCR purification Kit (Qiagen). PBS and LB1, LB2, LB3 and RIPA wash buffers 194 were all supplemented with Sodium Butyrate (Sigma), cOmplete EDTA-free protease inhibitor 195 cocktail (Roche) and PMSF (Sigma) immediately before use. Sequencing was performed on Illumina HiSeq v4 platform using 75-bp paired-end sequencing.

For qPCR, KAPA SYBR Fast qPCR kit was used following the manufacturer's protocol. \% Input was calculated as $100 \times 2^{\Delta \mathrm{Ct}}$, where $\Delta \mathrm{Ct}=\mathrm{Ct}$ Normalized input $-\mathrm{Ct}_{\mathrm{Chlp}} ; \mathrm{Ct}_{\text {Normalized input }}=\mathrm{Ct}$ Input $-\log _{2}$ (input dilution factor); input dilution factor = fraction of input saved relative to each IP $\mathrm{x}$ dilution of input for qPCR. Sequences of primers used in ChIP-qPCR are listed in Table S4.

202

\section{ChIP-seq data processing and analysis}

204 Reads from ChIP-seq samples and input were mapped to the human genome GRCh38 using 205 Burrows-Wheeler Aligner (BWA) version 0.7.17 (ref. 29) on default parameters. Duplicates were 206 marked by Samtools mkdup ${ }^{30}$. Peaks (broad and narrow) were called by MACS2 version 1.4.1 (ref. 207 31) using input DNA as control with parameters --broad-cutoff 0.01 for broad peaks, and -q 0.01 for 208 narrow peaks. Broad and narrow peaks were merged into a union set. Broad peaks that overlap with 209 one or more narrow peaks were removed. Locations of peaks (promoter, exonic, intronic or 210 intergenic) were computed by customized scripts using Ensembl transcript annotation of GRCh38 211 version 91. Peaks were considered to be associated with promoter(s) if more than a half of the peak 212 region was located within $\pm 2 \mathrm{~kb}$ from the transcription start site. Promoter occupancies of a 213 transcript were quantified as the highest MACS2 signal among all the peaks within the $2 \mathrm{~kb}$ window. 214 If multiple isoforms existed, genic promoter occupancies were calculated as the highest signal 215 among isoforms. Public data used was GSM1845161 and GSE83671. 


\section{RNA extraction and RNA-seq processing}

218 RNA was extracted from AML cells with RNeasy Plus Mini Kit (Qiagen) according to manufacturer's

219 instructions. Sequencing was performed on Illumina HiSeq v4 platform with 75-bp paired-end 220 sequencing.

221 RNA-seq reads were mapped to the human genome assembly GRCh38 using STAR version 6.43 (ref.

222 32), tolerating mismatch rate of 0.01 and allowing maximal intron lengths of 50kb. Read counts were

223 calculated by STAR using Ensembl annotation of GRCh38 version 91 and normalized as fragments per

224 kilobase of gene length per million uniquely mapped reads (FPKM). Differential expression analysis

225 was done by DESeq2 (ref. 33) using paired sample design and significant genes were identified using

226 adjusted $p$-values of 0.05 as a cut-off.

227

228 RNA-seq and ChIP-seq data are available from Gene Expression Omnibus under accession number

229 GSE133516 (Security Token, svobogacrrchtkd).

230

\section{Statistical analysis}

232 Student's $t$-test was used for statistical testing unless stated otherwise. Mean was calculated from at

233 least three replicates, as indicated in each figure, and error bars represent the standard deviation. $P$

234 values $\leq 0.05$ were considered statistically significant. 


\section{Results}

Loss of KAT7 induces myeloid differentiation and apoptosis in AML cell lines with MLL fusion oncoprotein.

We previously catalogued fitness genes in 5 AML cell lines (MOLM-13, MV4-11, OCI-AML2, OCIAML3 and HL-60) by genome-scale CRISPR-KO screening and characterized promising therapeutic targets including the histone lysine acetyltransferase KAT2A ${ }^{11}$. We further looked into genes encoding histone modifying enzymes and found that several histone acetyl transferases (HATs) were required for AML cell proliferation (Fig. 1A). Notably, two of the MYST family HATs, KAT6A and KAT7, were essential for AML cell lines driven by MLL-X fusion genes: MOLM-13 (driven by MLL-AF9), MV411 (MLL-AF4) and OCI-AML2 (MLL-AF6). In order to validate these fitness defects, we first investigated the effects on proliferation in MOLM-13 and MV4-11 by gRNA-mediated KO and found that KAT7 loss exhibited a consistent strong anti-proliferative effect in both cell lines (Fig. S1A). We then tested the impact of genetic disruption of KAT7 on proliferation of all cell lines used in our CRISPR-KO screens and 3 additional cell lines, and found that, consistent with MOLM-13 and MV411, KAT7 loss decreased the proliferation of other MLL-X cell lines, namely OCI-AML2, THP-1 (MLLAF9) and Nomo-1 (MLL-AF9), but not of MLL-WT cell lines such as OCl-AML3, HL-60 and K562 (Figs. $1 B$ and S1BC). The proliferation phenotype was associated with increased expression of the myeloid differentiation marker CD11b in MOLM-13 and OCI-AML2 (Fig. 1C). KAT7 loss also led to an increased apoptosis in MLL-X cell lines (Fig. 1D). Collectively, these results indicate that KAT7 is required for in vitro survival and proliferation of leukemic cells driven by $M L L-X$ fusion oncogenes. To assess if KAT7 inactivation has also anti-proliferative effects in vivo, we injected MOLM-13 cells previously transduced with a KAT7 gRNA (KAT7-KO) into NSGW41 male mice. Mice transplanted with KAT7-KO MOLM-13 cells showed significantly slower AML progression and increased survival, compared to those injected with MOLM-13 expressing a control gRNA (Fig. 1E, F), indicating the requirement of KAT7 for in vivo proliferation.

\section{KAT7 regulates global H3K14 and H4K12 acetylation}

KAT7 is known to form two distinct HAT complexes that acetylate either $\mathrm{H} 3$ or $\mathrm{H}^{23}$. We therefore investigated the acetylation status of $\mathrm{H} 3$ and $\mathrm{H} 4$ tails in KAT7 KO MOLM-13 cells. Among the potential KAT7 acetylation sites, acetylation of Lysine-14 residue of H3 (K3K14ac) and Lysine-12 of H4 (H4K12ac) were completely abolished upon KAT7 KO, whereas other acetylation sites were not affected (Fig. 2A). We additionally investigated major histone marks for active transcription, namely H3K4me3, H3K9ac and H3K27ac, and found that KAT7 loss did not affect the global level of these marks (Fig. 2B). Interestingly, global loss of H3K14ac and H4K12ac upon KAT7 loss was observed in 
270 all AML cell lines tested, irrespective of the MLL translocation status or proliferation phenotype (Fig.

2712 2).

272

273 Acetyltransferase activity of KAT7 is essential for leukemic maintenance

274 Since both MLL-X and MLL-WT AML cell lines showed globally reduced H3K14 and H4K12 acetylation

275 upon KAT7 KO but only MLL-X AML cell lines showed reduced proliferation, we considered that non-

276 catalytic roles of KAT7 complexes may have played a role in the observed proliferative defect. To

277 rule out such possibility, we performed cDNA reconstitution experiments using gRNA-resistant WT

278 and HAT-dead KAT7 cDNAs. For the HAT-dead KAT7 mutant, we utilized a previously characterized

279 E508Q MYST domain mutant ${ }^{34}$. To replace endogenous KAT7 with a cDNA-derived protein, we first

280 expressed a gRNA-resistant KAT7 CDNA and then knocked out the endogenous KAT7 by lentiviral

281 transduction of KAT7 gRNA. Western blot analyses showed that control (GFP only) MOLM-13 cells

282 lost KAT7 as well as acetylation marks on both H3K14 and H4K12 upon the endogenous KAT7 KO,

283 whereas cells carrying the WT KAT7 CDNA retained KAT7 protein and both acetylation marks, even

284 after gRNA-targeting of the endogenous KAT7 gene (Fig. 2D), indicating the appropriate

285 reconstitution of KAT7 activity. In contrast, cells expressing HAT-dead E508Q mutant KAT7 protein

286 completely lost both acetylation marks after endogenous KAT7 KO (Fig. 2D). We noted that cells

287 expressing both endogenous KAT7 and exogenous HAT-dead KAT7 showed considerably reduced

288 H4K12ac, although H3K14ac was maintained at the level similar to that of the control cells. This

289 suggest a dominant negative effect of HAT-dead KAT7 protein; however, proliferation of MOLM-13

290 cells expressing either exogenous KAT7 was not significantly different (Fig. 2E). We then analyzed

291 proliferation of KAT7-reconstituted cells and found that KAT7-WT-expressing cells did not show a

292 proliferation defect, ruling out the possibility that off-target effects of KAT7 gRNAs might have been

293 responsible for the observed phenotype (Fig. 2F, middle panel). In sharp contrast, the cells

294 expressing HAT-dead KAT7 showed proliferation defect(Fig. 2F, right panel), similar to that in

295 endogenous KAT7 KO cells. This proliferation defect was associated with increased CD11b expression

296 (Fig. 2G). Thus, the cells expressing HAT-dead KAT7 phenocopied the endogenous KAT7 KO cells in

297 histone acetylation, proliferation and differentiation. These results strongly indicate that KAT7-

298 mediated acetylation plays crucial roles in the maintenance of the leukemic program driven by the

299 MLL-X fusion protein.

300

301 KAT7 loss leads to down-regulation of a specific set of MLL-AF9 target genes

302 In order to analyze transcriptomic changes upon KAT7 KO, we performed RNA-seq analyses of cells

303 transduced with lentivirus expressing KAT7 or control gRNA. We chose MOLM-13 and OCI-AML3 as 
304 the MLL-X positive and negative cell lines, respectively, in this analysis. On day 3 after transduction,

305 we observed a total of 544 differentially expressed (DE) genes (317 up- and 227 down-regulation) in

306 MOLM-13 (Fig. 3A). The number of DE genes increased further on day 5 to 4093 (1954 up- and 2139

307 down-regulation). In contrast, the KAT7-independent OCI-AML3 showed only 11 genes on day 3 and

308501 on day 5. Consistent with myeloid differentiation observed by flow cytometry (CD11b

309 upregulation, Fig. 1C), gene set enrichment analysis (GSEA) revealed that a gene set consisting of

310 genes upregulated upon myeloid differentiation was significantly enriched on days 3 and 5 (Fig. 3B,

311 left). Concomitantly, the MLL-AF9 target gene set was significantly depleted on day 5 (Fig. 3B, center

312 bottom). Interestingly, although negative enrichment of the MLL-AF9 target gene set was not yet

313 detected on day 3 , enrichment of the myeloid differentiation-associated gene set was already

314 evident (Fig. 3B, top), indicating the rapid transcriptomic alteration upon KAT7 loss and thus key

315 roles of KAT7 in maintaining MLL-AF9-driven leukemic program.

316

317 Through inspection of differentially expressed genes in MOLM-13 on day 3 , we identified that a 318 small fraction of the MLL-AF9 target genes, most notably MEIS1, PBX3, JMJD1C, SENP6 and MEF2C, 319 were significantly down-regulated (Fig. 3C). Together with HOXA9, MEIS1 and PBX3 are known to 320 form a complex and promote transcription of HOXA target genes ${ }^{35}$. JMJD1C and MEF2C are also 321 known to play an important role in MLL-AF9-induced leukemogenesis ${ }^{36,37}$. In addition, these genes 322 have recently been shown to be among genes, of which MLL-AF9 binding is not limited to their 323 promoter region but spreads into their gene body ${ }^{38}$. These MLL-X oncoprotein "spreading" genes 324 are collectively more susceptible to DOT1L inhibition than MLL-X "non-spreading" genes ${ }^{38}$, 325 indicating that spreading genes comprise the core downstream network of the $M L L-X$-driven 326 leukemic program. We further performed GSEA on the day 3 expression profile with a gene set 327 consisting of protein-coding KAT7-bound MLL-AF9 spreading genes (see below, Table S1). Although 328 enrichment of the comprehensive MLL-AF9 target gene set was not detected on day 3 (Fig. 3B, 329 center), this smaller subset was significantly depleted (Fig. 3B, right). Enrichment was not observed 330 in the MLL-WT, KAT7-independent OCI-AML3 cell line. These results indicate that KAT7 loss disrupts 331 a leukemia maintenance program through down-regulation of the MLL-AF9 spreading genes, rather 332 than the entirety of MLL-AF9 target genes.

\section{KAT7 binds to promoters of active genes, especially a subset of MLL-AF9 spreading genes}

335 We next investigated the genomic location of KAT7 binding sites using chromatin336 immunoprecipitation followed by sequencing (ChIP-seq) analysis. We again chose MOLM-13 and 337 OCI-AML3 for this analysis and identified 9214 and 60153 KAT7 peaks, respectively (Fig. 4A). KAT7 
338 was most enriched at promoters in both cell lines; $63.3 \%$ of the KAT7 signals in MOLM-13 and 48.5

$339 \%$ in OCl-AML3 were found in promoter regions ( $\pm 2 \mathrm{~kb}$ from TSSs) (Fig. 4A, B, D). Consistent with 340 previously reported ChIP-seq studies ${ }^{16,23,24}$, KAT7 occupancy in the AML cell lines is significantly 341 correlated with the expression levels of target genes (Fig. 4B-E). We then focused our analysis on 342 the MLL-AF9 spreading gene set. These genes are expressed not only in the MLL-AF9+ve MOLM-13 343 but also in MLL-AF9-ve OCI-AML3 with a significant correlation of their expression levels between 344 the two cell lines (Fig. $4 \mathrm{~F}, \mathrm{r}^{2}=0.80$ ). As KAT7 marks expressed genes, the majority of the MLL-AF9 345 spreading genes were bound by KAT7 in both cell lines (Fig. 4G). We then compared expression 346 levels and KAT7 promoter occupancy of the spreading genes (Fig. $4 \mathrm{H}, \mathrm{I}$ ) and found that, consistent 347 with the genome-wide patterns, OCI-AML3 showed good correlation between the two parameters 348 (Fig. 4I). However, MOLM-13 did not show clear correlation, but KAT7 rather seemed to be highly 349 bound to a small fraction of the MLL-AF9 spreading genes (Fig. 4H). We further compared KAT7 350 promoter occupancy with expression fold changes on day 3 after KAT7 KO and found that in MOLM35113 genes with higher KAT7 occupancy were more susceptible to and down-regulated upon KAT7 loss 352 (Fig. 4J). In particular, MEIS1, PBX3, JMJD1C and SENP6, which were significantly down-regulated on 353 day 3 (Fig. 3C), showed particularly high KAT7 promoter occupancy (Fig. 3J). There were significant 354 differences in KAT7 promoter occupancy between the down-regulated and the up-regulated $(n=4)$ or 355 not-differentially expressed ( $n=126)$ genes (Fig. 3K). In sharp contrast and consistent with its KAT7 356 independence, in OCI-AML3 KAT7 loss did not show a significant impact on expression on day 3, 357 even for genes with KAT7 promoter occupancy comparable to those down-regulated in MOLM-13 358 (Fig. 4L, M). In both cell lines, for non-MLL-AF9 target genes, there was no significant difference in 359 KAT7 promoter occupancy between up-regulated, down-regulated and not-differentially expressed 360 genes (Fig. S2A-D). Taken together, these results suggest that although KAT7 is not required for 361 general transcription in AML, KAT7 and its acetylase activity are required for MLL-AF9 oncoprotein to 362 sufficiently up-regulate and maintain expression of a specific set of the MLL-AF9 spreading genes. MLL-fusion associated machineries are evicted from their target loci upon KAT7 loss

365 To study the consequences of KAT7 loss at the chromatin level, we employed the auxin-inducible 366 degron (AID) system ${ }^{39}$. For this, we first stably expressed KAT7-AID and then knocked out the 367 endogenous KAT7 with gRNA. We confirmed that proliferation of KAT7-AID MOLM-13 was similar to 368 that of parental MOLM-13 cells (Fig. S3A), indicating that AID-tagged KAT7 is functional. We then 369 introduced OsTIR1 and treated cells with auxin indole-3-acetic acid (IAA) to deplete KAT7 protein. 370 The KAT7 protein level rapidly decreased and plateaued $2 \mathrm{~h}$ after treatment (Fig. 5A). This was 371 associated with complete loss of H3K14ac over the same time course (Fig. 5A) and followed by 
372 myeloid differentiation (CD11b) after $48 \mathrm{~h}$ (Fig. 5B). Therefore, the phenotype after IAA treatment

373 faithfully mirrored those seen with CRISPR-mediated KAT7 KO (Figs. $1 \mathrm{C}$ and 2A), indicating that the

374 KAT7-AID system can be used to study the direct effects of KAT7 loss.

375

376 Using this degron system, we first investigated whether KAT7 is required for MLL-AF9 fusion protein

377 to maintain its binding to the promoters of key MLL-AF9 spreading genes, namely JMJD1C, SENP6,

378 PBX3 and MEIS1, which were down-regulated significantly on day 3 after KAT7 KO (Fig. 3C) and 379 bound by KAT7 with very high occupancy (Fig. 4J). ChIP followed by quantitative PCR (ChIP-qPCR) 380 analysis using antibodies against MLL N-terminal and AF9 C-terminal regions $24 \mathrm{~h}$ after IAA 381 treatment showed no significant changes in their occupancy at the promoter region (Fig. S3B). This 382 observation is consistent with previous findings that MLL-AF9 recruitment is dependent on interactions with MENIN ${ }^{40}$, LEDGF $^{41}$ and the PAF complex ${ }^{42}$.

385 We next investigated the binding of the histone acetylation "reader" BRD4 at gene promoters. BRD4 386 is a key interactor of MLL-fusion proteins and a target of anti-leukemic drugs ${ }^{5,6}$. Globally, BRD4 387 occupancy at promoter regions is highly correlated with KAT7 occupancy (Fig. 5C). At the selected 388 key MLL-AF9 spreading genes, KAT7, MLL-AF9 and BRD4 highly co-localized (Fig. 5D). Upon KAT7 389 depletion, BRD4 markedly dissociated from the promoters of the key spreading genes (Fig. 5E). This 390 was associated with dissociation of AFF1, a scaffold protein of the super elongation complex (SEC), 391 from these promoters (Fig. 5F). In addition, RNA polymerase II Serine 5 phosphorylation (PollI-pS5) 392 occupancy was also dramatically reduced at these loci (Fig. 5G). These results suggest that KAT7393 mediated histone acetylation serves as a scaffold for BRD4 binding to chromatin, which is required 394 for sustained recruitment of transcriptional activators (Fig. 5H). 
396

397

398

399

400

401

402

403

404

405

406

407

408

409

410

411

412

413

414

415

416

417

418

419

420

421

422

423

424

425

426

427

428

429

\section{Discussion}

Despite advances in understanding its genomics and molecular pathogenesis, AML remains lethal to the majority of sufferers ${ }^{43}$ and anti-AML mainstream therapies have not changed significantly for several decades. Among different AML subtypes, cases driven by $M L L-X$ fusion genes continue to represent a poor prognostic category ${ }^{1-3}$ and despite recent developments in the field ${ }^{5,6,8,10,44}$ clinical progress is still lacking, emphasizing the need for new therapies. Here, we demonstrate that KAT7 represents a promising novel therapeutic target for MLL-X AML and provide insights into its function in the maintenance of these leukemias.

KAT7-containing complexes have been shown to acetylates lysine residues of histone $\mathrm{H} 3$ and $\mathrm{H} 4$ tails. Specificity for the target histone is determined by the scaffold subunits, BRPF and JADE. In in vitro HAT assays using nucleosomes, BRPF-containing complex acetylates H3K14 and K23, while JADE-containing complex acetylates H4K5, K8 and K12 (ref. 23). These scaffold subunits can also form a HAT complex with KAT6A and KAT6B and show a similar specificity against H3 and H4 (ref. 23). In principle, this complex formation is interchangeable, but seems to be differentially regulated in different cell types. For example, in HeLa cells, purified KAT7-containing HAT complex contained JADE but not BRPF ${ }^{27}$, and BRPF-containing HAT complexes predominantly contain KAT6A/B ${ }^{23}$. In keeping with this, knock-down of KAT7 in HeLa cells results in reduction of all of the three acetylation marks on $\mathrm{H} 4$ (ref. 27). In contrast, KAT7-KO in mouse embryonic fibroblasts and KAT7 knock-down in erythroblasts led to reduction specifically of H3K14ac among the 5 potential acetylation sites and had no effect on histone $\mathrm{H} 4$ (ref. 24,25). In the present study, we found that KAT7 KO in AML cells resulted in complete loss of both H3K14ac and H4K12ac, a pattern that has not been described before. This strongly suggests that KAT7 is solely responsible for acetylation of these 2 sites and that KAT6A does not compensate for KAT7 loss. It has been shown that in mouse embryos KAT6A plays a specific role in regulating local H3K9 acetylation at the Hoxa and Hoxb loci and is not involved in global H3K9 and H3K14 acetylation ${ }^{45}$. This might be the case in AML cells, indicating that KAT6A and KAT7 play non-overlapping roles in histone acetylation. KAT6A also showed fitness defects in MLL-X AML cell lines in our analysis. It would be interesting to investigate the molecular basis of KAT6A dependency and the mechanisms by which cell-type-specific complex formation is regulated.

Besides the general correlation between expression level and KAT7 promoter occupancy, we found that KAT7 strongly bound to a small subset of MLL-AF9 spreading genes in MOLM-13. The expression of this subset is especially susceptible to KAT7 loss and it includes well-known leukemia maintenance 
430 genes in MLL-X-driven AML such as MEIS1, PBX3, JMJD1C and MEF2C. MLL-X target genes are known

431 to be associated with high level of H3K79me2, which is deposited by histone methyltransferase

432 DOT1L $L^{8,44}$. In MLL-X spreading genes, H3K79me2 marks also spread into gene bodies, showing

433 broader peaks ${ }^{38}$. These spreading genes were shown to be more sensitive to DOT1L inhibition than

434 the other genes (non-spreading and no-bound genes); the entire set of the spreading genes are

435 downregulated upon DOT1L inhibition ${ }^{38}$. This pattern of down-regulation is in sharp contrast to the

436 case of KAT7 loss, in which only a subset of the spreading genes that are highly bound by KAT7 are

437 down-regulated. Therefore, recruitment of KAT7 complex to promoters of this subset might be regulated differently from the rest of the promoters.

439

440 As a potential reader of H3K14ac and/or H4K12ac by KAT7, we investigated BRD4 and showed that

441 BRD4 dissociated from the promoter of the key spreading genes upon KAT7 loss. BRD4 is known to 442 play crucial roles in maintaining $M L L-X$-driven leukemic programs ${ }^{5,6}$. A prominent role of BRD4 in $443 \mathrm{AML}$ is to activate super-enhancers at the MYC locus and maintain high MYC expression ${ }^{46}$. 444 Bromodomain inhibitors such as JQ1 and i-BET151 disrupt the interaction between BRD4 and 445 acetylated histones, and evict BRD4 from chromatin, thereby leading to rapid downregulation of 446 MYC. In addition to the role at enhancers, BRD4 is known to play a role in promoting Pol II 447 elongation at promoter-proximal regions by recruiting SEC including P-TEFb ${ }^{46}$. Accordingly, we 448 observed the reduced binding of AFF1 scaffold protein of SEC upon KAT7 loss. This suggests that Pol 449 II elongation is severely affected, causing downregulation of target genes. However, since the role of 450 BRD4 at promoters is generic, it is still unclear why only a small subset of MLL-AF9 spreading genes 451 are affected by KAT7 loss in MOLM-13 and why gene expression is relatively stable, even in the 452 absence of KAT7, in the KAT7-independent OCI-AML3 cells. It is plausible that additional acetylated 453 histone readers are involved in KAT7-dependent transcriptional activation for the MLL-AF9 454 spreading genes. From this perspective, it is interesting to observe that pS5-Pol II reduced its 455 occupancy at the selected promoters. Serine 5 of Pol II is phosphorylated by TFIIH after Pol II loading 456 onto promoters ${ }^{47}$. Subsequent Serine 2 phosphorylation on the C-terminal domain of Pol II by P457 TEFb promotes Pol II elongation ${ }^{47}$. Therefore, KAT7-mediated histone acetylation may also be 458 required for the initiation phase of Pol II transcription. Further studies are required to elucidate the 459 molecular basis of KAT7-dependent transcription of MLL-AF9 target genes.

461 Collectively, our findings reveal that KAT7 acts upstream of BRD4 to recruit MLL-fusion associated 462 machineries to the promoter of key MLL-AF9 target genes, which represent an alternative 463 therapeutic target for $M L L-X$ leukemia. We anticipate that our work will motivate the development 
bioRxiv preprint doi: https://doi.org/10.1101/2020.04.25.054049; this version posted April 25, 2020. The copyright holder for this preprint (which

was not certified by peer review) is the author/funder, who has granted bioRxiv a license to display the preprint in perpetuity. It is made available under aCC-BY-NC-ND 4.0 International license.

464 of small-molecule inhibitors that are specific to KAT7 and highlight KAT7 as a potential therapeutic 465 target for MLL-fusion AML. 


\section{Acknowledgements}

467 This work was funded by the Wellcome Trust (WT206194), the Kay Kendall Leukaemia Fund 468 (KKL920), Bloodwise (17006) and Exonate Ltd. K.T. was funded by a Wellcome Trust Sir Henry 469 Wellcome Fellowship (grant reference RG94424). G.S.V. was funded by a Cancer Research UK Senior 470 Cancer Fellowship (C22324/A23015) and a Wellcome Trust Senior Fellowship in Clinical Science 471 (WT095663MA). We thank Bee Ling Ng, Jennifer Graham, Christopher Hall and Sam Thompson from 472 the Wellcome Sanger Institute Cytometry Core Facility team for help with flow cytometry. We are 473 grateful to the staff of the Sanger Institute Research Support Facility for help with mouse 474 experiments and the staff of the Sanger Institute Core Sequencing facility for sequencing. We thank 475 Mathew Garnett for providing the Nomo-1 cell line; Pedro for his help compiling figures using Adobe 476 Illustrate and Josep Nomdedeu for help advices in writing the manuscript.

477

478

Authors contributions

479 K.Y. conceived the study and designed the experiments. Y.Z.A performed the experiments and 480 analyzed the data. E.D.B. performed and analyzed the in vivo mouse studies. M.G. and S.H.O. 481 conducted bioinformatic analyses. J.Y., B.J.P.H., M.G., and K.T. helped with data interpretation and 482 direction. M. Gozdecka and X.C. advised on ChIP experiments. Y.Z.A., M.G., J.Y., G.S.V. and K.Y. wrote 483 the manuscript. All authors reviewed the manuscript.

484

\section{Competing interests}

486 G.S.V. is a consultant for Kymab and Oxstem.

\section{References}

1 Papaemmanuil E, Gerstung M, Bullinger L, Gaidzik VI, Paschka P, Roberts ND et al. Genomic Classification and Prognosis in Acute Myeloid Leukemia. N Engl J Med 2016; 374: 2209-2221. doi:10.3390/jcm5030033. cell development. Nat Rev Cancer 2007; 7: 823-833. 4 Winters AC, Bernt KM. MLL-Rearranged Leukemias-An Update on Science and Clinical Approaches. Front Pediatr 2017; 5: 4.

5 Dawson MA, Prinjha RK, Dittmann A, Giotopoulos G, Bantscheff M, Chan W-I et al. Inhibition of BET recruitment to chromatin as an effective treatment for MLL-fusion leukaemia. Nature 2011; 478: 529-533.

6 Zuber J, Shi J, Wang E, Rappaport AR, Herrmann H, Sison EA et al. RNAi screen identifies Brd4 
501 as a therapeutic target in acute myeloid leukaemia. Nature 2011; 478: 524-528.

502

503

504

505

506

507

508

509

510

511

512

513

514

515

516

517

518

519

520

521

522

523

524

525

526

527

528

529

530

531

532

533

534

535

536

537

538

539

7 Berthon C, Raffoux E, Thomas X, Vey N, Gomez-Roca C, Yee K et al. Bromodomain inhibitor OTX015 in patients with acute leukaemia: a dose-escalation, phase 1 study. Lancet Haematol. 2016; 3: e186--95. .

8 Daigle SR, Olhava EJ, Therkelsen CA, Majer CR, Sneeringer CJ, Song J et al. Selective killing of mixed lineage leukemia cells by a potent small-molecule DOT1L inhibitor. Cancer Cell 2011; 20: 53-65.

9 Daigle SR, Olhava EJ, Therkelsen CA, Basavapathruni A, Jin L, Boriack-Sjodin PA et al. Potent inhibition of DOT1L as treatment of MLL-fusion leukemia. Blood 2013; 122: 1017-1025.

10 Grembecka J, He S, Shi A, Purohit T, Muntean AG, Sorenson RJ et al. Menin-MLL inhibitors reverse oncogenic activity of MLL fusion proteins in leukemia. Nat Chem Biol 2012; 8: 277-284.

11 Tzelepis K, Koike-Yusa H, De Braekeleer E, Li Y, Metzakopian E, Dovey OM et al. A CRISPR Dropout Screen Identifies Genetic Vulnerabilities and Therapeutic Targets in Acute Myeloid Leukemia. Cell Rep 2016; 17: 1193-1205.

12 Wang T, Yu H, Hughes NW, Liu B, Kendirli A, Klein K et al. Gene Essentiality Profiling Reveals Gene Networks and Synthetic Lethal Interactions with Oncogenic Ras. Cell 2017; 168: 890903.e15.

13 Tzelepis K, De Braekeleer E, Aspris D, Barbieri I, Vijayabaskar MS, Liu W-H et al. SRPK1 maintains acute myeloid leukemia through effects on isoform usage of epigenetic regulators including BRD4. Nat Commun 2018; 9: 5378.

14 Sheikh BN, Akhtar A. The many lives of KATs - detectors, integrators and modulators of the cellular environment. Nat Rev Genet 2019; 20: 7-23.

15 Voss AK, Thomas T. MYST family histone acetyltransferases take center stage in stem cells and development. Bioessays 2009; 31: 1050-1061.

16 Avvakumov N, Côté J. The MYST family of histone acetyltransferases and their intimate links to cancer. Oncogene 2007; 26: 5395-5407.

17 Carapeti M, Aguiar RC, Goldman JM, Cross NC. A novel fusion between MOZ and the nuclear receptor coactivator TIF2 in acute myeloid leukemia. Blood 1998; 91: 3127-3133.

18 Kitabayashi I, Aikawa Y, Yokoyama A, Hosoda F, Nagai M, Kakazu N et al. Fusion of MOZ and p300 histone acetyltransferases in acute monocytic leukemia with a $t(8 ; 22)(p 11 ; q 13)$ chromosome translocation. Leukemia 2001; 15: 89-94.

19 Panagopoulos I, Fioretos T, Isaksson M. Fusion of the MORF and CBP genes in acute myeloid leukemia with the $\mathrm{t}(10 ; 16)(\mathrm{q} 22 ; \mathrm{p} 13)$. Molecular Genetics 2001.https://academic.oup.com/hmg/article-abstract/10/4/395/2901550.

20 Esteyries S, Perot C, Adelaide J, Imbert M, Lagarde A, Pautas C et al. NCOA3, a new fusion partner for MOZ/MYST3 in M5 acute myeloid leukemia. Leukemia 2008; 22: 663-665.

21 Borrow J, Stanton VP Jr, Andresen JM, Becher R, Behm FG, Chaganti RS et al. The translocation $\mathrm{t}(8 ; 16)(\mathrm{p} 11 ; \mathrm{p} 13)$ of acute myeloid leukaemia fuses a putative acetyltransferase to the CREB-binding protein. Nat Genet 1996; 14: 33-41. 
540

22 Valerio DG, Xu H, Chen C-W, Hoshii T, Eisold ME, Delaney C et al. Histone Acetyltransferase Activity of MOF Is Required for MLL-AF9 Leukemogenesis. Cancer Res 2017; 77: 1753-1762.

23 Lalonde M-E, Avvakumov N, Glass KC, Joncas F-H, Saksouk N, Holliday M et al. Exchange of associated factors directs a switch in $\mathrm{HBO} 1$ acetyltransferase histone tail specificity. Genes Dev 2013; 27: 2009-2024.

24 Mishima Y, Miyagi S, Saraya A, Negishi M, Endoh M, Endo TA et al. The Hbo1-Brd1/Brpf2 complex is responsible for global acetylation of H3K14 and required for fetal liver erythropoiesis. Blood. 2011; 118: 2443-2453.

25 Kueh AJ, Dixon MP, Voss AK, Thomas T. HBO1 is required for H3K14 acetylation and normal transcriptional activity during embryonic development. Mol Cell Biol 2011; 31: 845-860.

26 Feng $\mathrm{Y}$, Vlassis A, Roques $\mathrm{C}$, Lalonde $\mathrm{M-E}$, González-Aguilera C, Lambert J-P et al. BRPF3HBO1 regulates replication origin activation and histone H3K14 acetylation. EMBO J 2016; 35: 176-192.

27 Doyon Y, Cayrou C, Ullah M, Landry A-J, Côté V, Selleck W et al. ING tumor suppressor proteins are critical regulators of chromatin acetylation required for genome expression and perpetuation. Mol Cell 2006; 21: 51-64.

28 Hodgkins A, Farne A, Perera S, Grego T, Parry-Smith DJ, Skarnes WC et al. WGE: a CRISPR database for genome engineering. Bioinformatics 2015; 31: 3078-3080.

29 Li H, Durbin R. Fast and accurate short read alignment with Burrows-Wheeler transform. Bioinformatics. 2009; 25: 1754-1760.

$30 \mathrm{Li} \mathrm{H}$, Handsaker B, Wysoker A, Fennell T, Ruan J, Homer N et al. The Sequence Alignment/Map format and SAMtools. Bioinformatics 2009; 25: 2078-2079.

31 Zhang Y, Liu T, Meyer CA, Eeckhoute J, Johnson DS, Bernstein BE et al. Model-based analysis of ChIP-Seq (MACS). Genome Biol 2008; 9: R137.

32 Dobin A, Davis CA, Schlesinger F, Drenkow J, Zaleski C, Jha S et al. STAR: ultrafast universal RNA-seq aligner. Bioinformatics 2013; 29: 15-21.

33 Love MI, Huber W, Anders S. Moderated estimation of fold change and dispersion for RNAseq data with DESeq2. Genome Biol 2014; 15: 550.

34 Foy RL, Song IY, Chitalia VC, Cohen HT, Saksouk N, Cayrou C et al. Role of Jade-1 in the histone acetyltransferase (HAT) HBO1 complex. J Biol Chem 2008; 283: 28817-28826.

35 Thorne RMW, Milne TA. Dangerous liaisons: cooperation between Pbx3, Meis1 and Hoxa9 in leukemia. Haematologica 2015; 100: 850-853.

36 Tarumoto Y, Lu B, Somerville TDD, Huang Y-H, Milazzo JP, Wu XS et al. LKB1, Salt-Inducible Kinases, and MEF2C Are Linked Dependencies in Acute Myeloid Leukemia. Mol Cell 2018; 69: 1017-1027.e6.

37 Chen M, Zhu N, Liu X, Laurent B, Tang Z, Eng R et al. JMJD1C is required for the survival of acute myeloid leukemia by functioning as a coactivator for key transcription factors. Genes Dev 2015; 29: 2123-2139. 
578

38 Kerry J, Godfrey L, Repapi E, Tapia M, Blackledge NP, Ma H et al. MLL-AF4 Spreading Identifies Binding Sites that Are Distinct from Super-Enhancers and that Govern Sensitivity to DOT1L Inhibition in Leukemia. Cell Rep 2017; 18: 482-495.

39 Nishimura K, Fukagawa T, Takisawa H, Kakimoto T, Kanemaki M. An auxin-based degron system for the rapid depletion of proteins in nonplant cells. Nat Methods 2009; 6: 917-922.

40 Yokoyama A, Somervaille TCP, Smith KS, Rozenblatt-Rosen O, Meyerson M, Cleary ML. The menin tumor suppressor protein is an essential oncogenic cofactor for MLL-associated leukemogenesis. Cell 2005; 123: 207-218.

41 Yokoyama A, Cleary ML. Menin critically links MLL proteins with LEDGF on cancer-associated target genes. Cancer Cell 2008; 14: 36-46.

42 Muntean AG, Tan J, Sitwala K, Huang Y, Bronstein J, Connelly JA et al. The PAF complex synergizes with MLL fusion proteins at HOX loci to promote leukemogenesis. Cancer Cell 2010; 17: 609-621.

43 Ferrara F, Schiffer CA. Acute myeloid leukaemia in adults. Lancet 2013; 381: 484-495.

44 Bernt KM, Zhu N, Sinha AU, Vempati S, Faber J, Krivtsov AV et al. MLL-rearranged leukemia is dependent on aberrant H3K79 methylation by DOT1L. Cancer Cell 2011; 20: 66-78.

45 Voss AK, Collin C, Dixon MP, Thomas T. Moz and retinoic acid coordinately regulate H3K9 acetylation, Hox gene expression, and segment identity. Dev Cell 2009; 17: 674-686.

46 Shi J, Vakoc CR. The mechanisms behind the therapeutic activity of BET bromodomain inhibition. Mol Cell 2014; 54: 728-736.

47 Brookes E, Pombo A. Modifications of RNA polymerase II are pivotal in regulating gene expression states. EMBO Rep 2009; 10: 1213-1219. 


\section{Figure 1. Loss of KAT7 exhibits anti-leukemic effects in vitro and in vivo}

602 A) Fitness effect of HATs from genome-scale CRISPR-KO screening in AML and non-AML cell lines ${ }^{11}$ 603 based on false discovery rate (FDR) values. B) Proliferation of KAT7 KO using two sgRNAs or empty 604 control in AML cell lines. Percentages of BFP-positive (KAT7-KO) cells were assayed at the indicated 605 time point and were normalized to day 4. Data are shown as mean \pm SD $(n=3)$. C) CD11b staining in 606 WT (empty gRNA) and KAT7 KO (gKAT7-A10) cells 7 days post transduction. D) Annexin V/PI staining 607 of WT (empty) and KAT7 KO (gKAT7-A10) cells 9 days post transduction. Data are shown as mean \pm 608 SD ( $\mathrm{n}=3)$. Two-tailed $t$-test was performed (N.S., not significant; $* *, P<0.01 ; * * *, P<0.001)$. E-F) 609 Xenograft analysis of KAT7-KO MOLM-13. Bioluminescent imaging (E) and Kaplan-Meier survival 610 analysis (F) were performed ( $n=5$ in each arm).

611

612

613 Figure 2. Catalytic activity of KAT7 is required for leukemic maintenance.

614 A-B) Western blot analyses of potential KAT7 acetylation sites on $\mathrm{H} 3$ and $\mathrm{H} 4$ (A) and major histone 615 marks associated with active transcription (B) in KAT7-KO cells. C) Western blot analysis of KAT7616 mediated acetylation on H3K14 and H4K12 in an additional MLL-AF9+ve cell line, THP-1, and MLL617 WT OCI-AML3 and HL-60. D) Western blot analysis of MOLM-13 expressing exogenous WT-KAT7 or 618 HAT-dead KAT7 (E508Q) with or without endogenous KAT7 KO by lentiviral gRNA expression. E) 619 Proliferation of MOLM-13 cells expressing exogenous KAT7 WT or E508Q mutant as well as 620 endogenous KAT7. F-G) Proliferation (F) and CD11b staining (G) of MOLM-13 expressing exogenous 621 KAT7 WT or E508Q mutant with endogenous KAT7 disrupted by gKAT7.

624 A) Numbers of differentially expressed (DE) genes in MOLM-13 and OCI-AML3 on day 3 and day 5 625 after gRNA-mediated KAT7 KO. B) GSEA on day 3 (top) and day 5 (bottom) transcriptomes in MOLM626 13. Gene sets consisting of genes upregulated upon myeloid differentiation (left), MLL-AF9 target 627 genes (centre), or MLL-AF9 spreading genes (right; Table S1) were used. C) MA plot of day 3 MOLM62813 comparing KAT7 WT and KO. Black, DE genes (adj.P<0.05); yellow, MLL-AF9 spreading genes. Key 629 MLL-AF9 spreading genes, namely MEIS1, PBX3, JMJD1C, SENP6 and MEF2C were highlighted.

Figure 4. KAT7 binds to and is required for expression of a subset of MLL-AF9 targets.

632 A) Distribution of KAT7 signals at promoters (TSS $\pm 2 \mathrm{~kb}$ ), exons (excluding promoter exons), introns 633 and intergenic regions quantified by MACS2 peaks. B) Average occupancy of KAT7 per gene per base 634 of highly active genes (>10 FPKM), active genes $(>0, \leq 10 \mathrm{FPKM})$ and inactive genes (FPKM $=0)$ in 
635 MOLM-13. C) Box plots showing KAT7 promoter occupancy in highly active, active and inactive 636 genes. Highly active genes showed significantly higher KAT7 promoter occupancy than active genes $637\left(P=3.5 \times 10^{-227}\right)$ and inactive genes $\left(P=5.0 \times 10^{-324}\right)$ by Mann-Whitney-Wilcoxon test. D-E) Same as 638 in B-C for OCl-AML3. Highly active genes showed significantly higher KAT7 promoter occupancy than 639 active genes and inactive genes by Mann-Whitney-Wilcoxon test. F) Comparison of expression level 640 of MLL-AF9 spreading genes between MOLM-13 and OCI-AML3. High correlation $\left(r^{2}=0.80\right)$ was 641 observed. Expression data described in ref. 11 were used. G) Significant overlap between KAT7642 bound and MLL-AF9-bound spreading genes in MOLM-13 $\left(P=3.9 \times 10^{-34}\right)$ and OCI-AML3 $(P=4.8 \times$

$64310^{-15}$ ) by Fisher's Exact test. $\left.\mathbf{H}-\mathbf{I}\right)$ Comparison between expression and KAT7 promoter occupancy for 644 MLL-AF9 spreading genes in MOLM-13 (H) and OCI-AML3 (I). Expression data described in ref. 11 645 were used. J-M) Comparison between KAT7 promoter occupancy and gene expression changes 3 646 days after KAT7 KO in MOLM-13 (J-K) and OCI-AML3 (L-M) for MLL-AF9 spreading genes. DE genes 647 are highlighted in blue (up) or red (down). Down-regulated genes show significantly higher KAT7 648 promoter occupancy, compared to up-regulated $(P=0.0027)$ and not differentially expressed genes $649\left(P=2.7 \times 10^{-7}\right)$ in MOLM-13 (K) but not in OCI-AML3 (M). One-tailed $t$-test was performed.

650

651 Figure 5. KAT7-dependent recruitment of BRD4 and SEC complex to a subset of MLL-AF9 spreading 652 genes.

653 A) Time-course western blot analyses of KAT7 and H3K14ac in IAA-treated KAT7-AID MOLM-13 cells.

654 B) FACS analysis of IAA-treated KAT7-AID MOLM-13 at $24 \mathrm{~h}$ using CD11b antibody. C) Genome-wide 655 correlation of KAT7 and BRD4 binding at promoter. D) Co-localization of KAT7, MLL-AF9 and BRD4 at 656 the promoter of the key MLL-AF9 spreading genes. E-G) ChIP-qPCR analysis of BRD4, AFF1 and pS5 657 Pol II after 24 hours of IAA treatment at the promoter region of the key MLL-AF9 spreading genes. 658 Data are shown as mean \pm S.D. $(n=3)$. Two-tailed t-test was performed $\left(*, P \leq 0.05 ;{ }^{* *}, P \leq 0.01 ;{ }^{* * *}\right.$, $659 P \leq 0.001)$. H) Model of KAT7-dependent recruitment of BRD4 and SEC complex machinery to drive 660 transcription of MLL-AF9 target genes. 
Figure 1

bioRxiv preprint doi: https://doi.org/10.1101/2020.04.25.054049; this version posted April 25, 2020. The copyright holder for this preprint (which was not certified by peer review) is the autho/funder, who has granted bioRxiv a license to display the preprint in perpetuity. It is made A
MLL-r
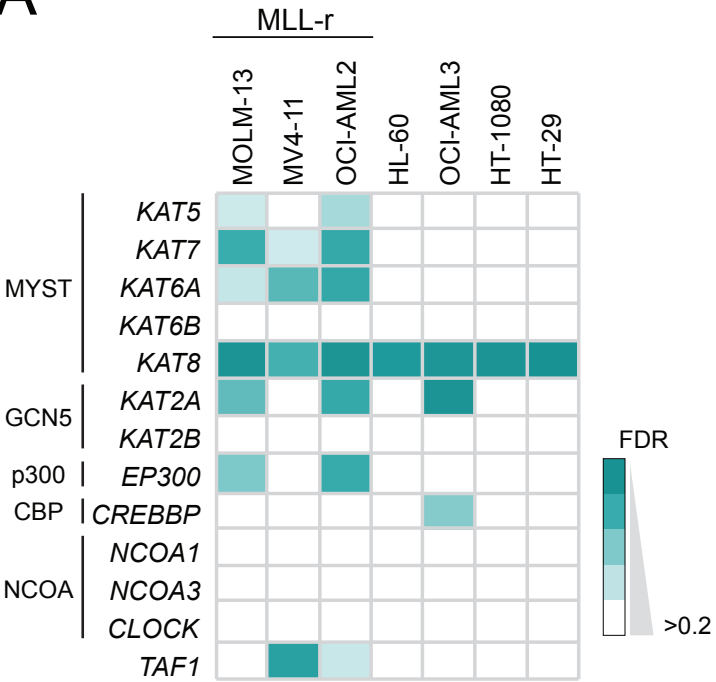

B
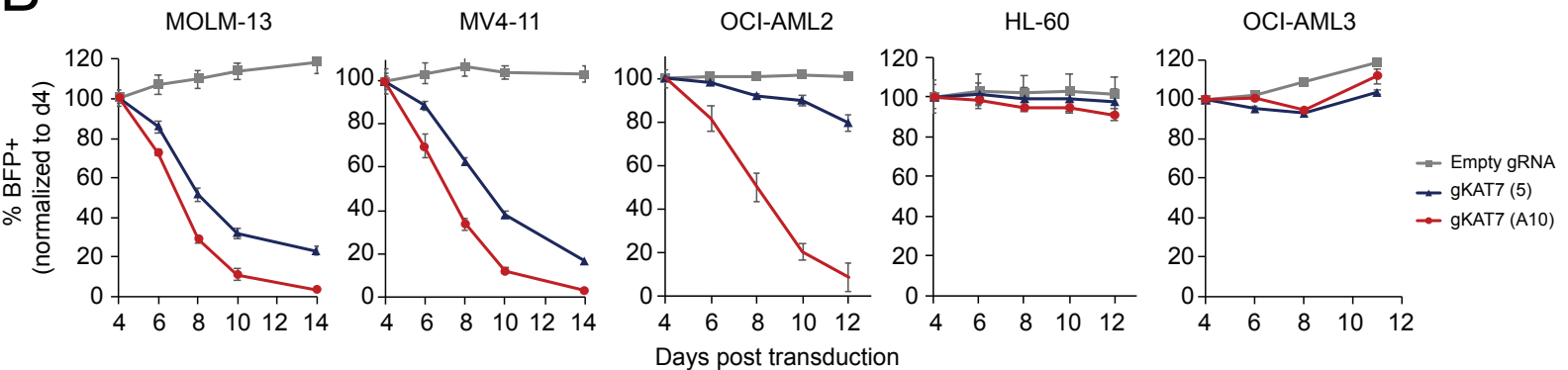

C
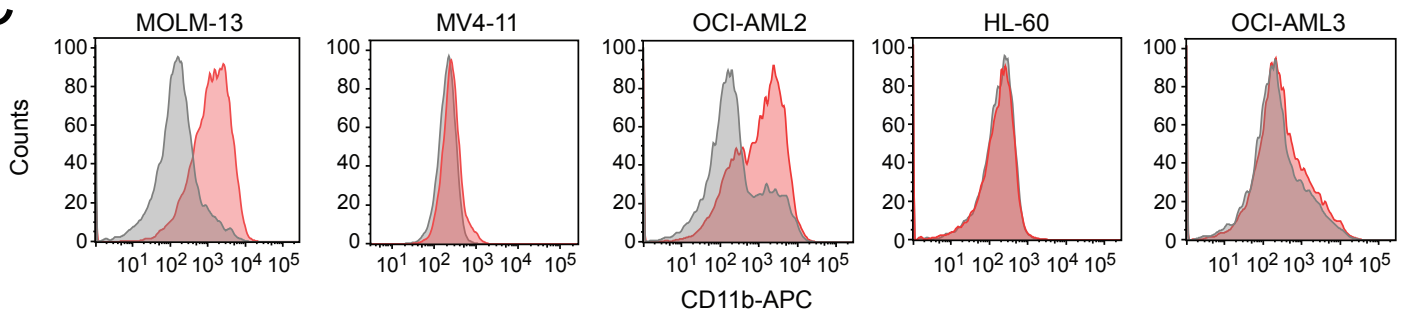

Empty gRNA gKAT7 (A10)

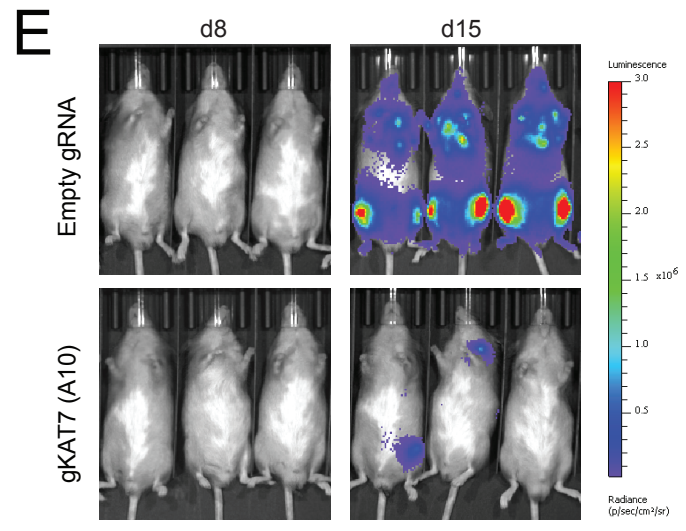

$\mathrm{F}$

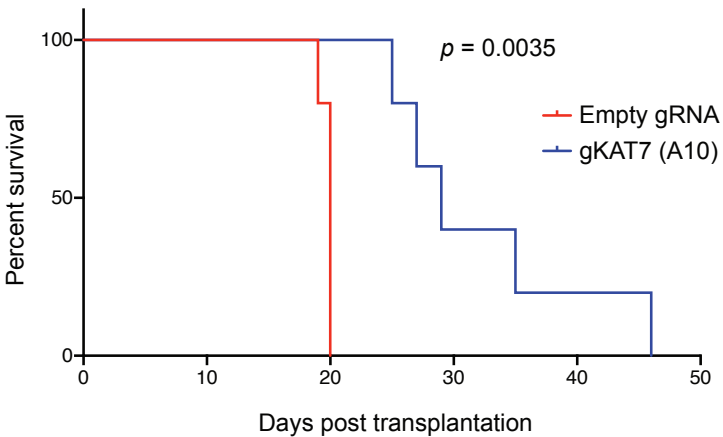


Figure 2

bioRxiv preprint doi: https://doi.org-30.1101/2020.04.25.054049; this vewsion posted April 25, 2020. The copyright holder for this preprint (which was not certified by peer reviem is the author/funder, who has grented bioRxiv a license to display the preprint in perpetuity. It is made
gKAT7 (A10) gKAT7 (A10)
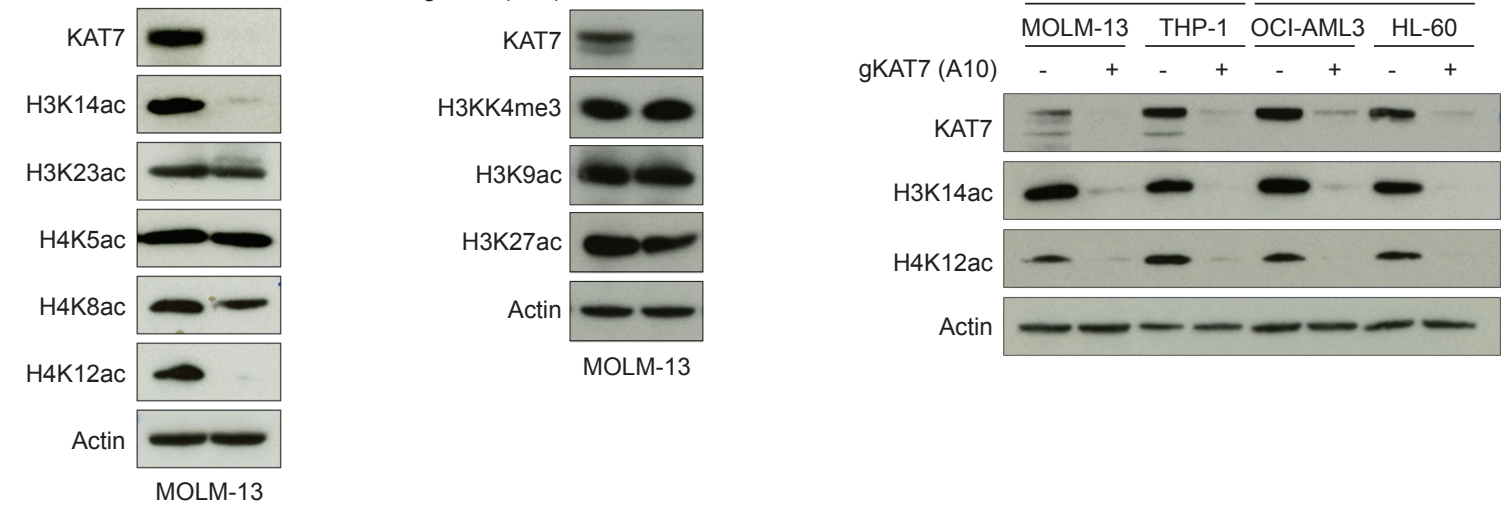

$D$

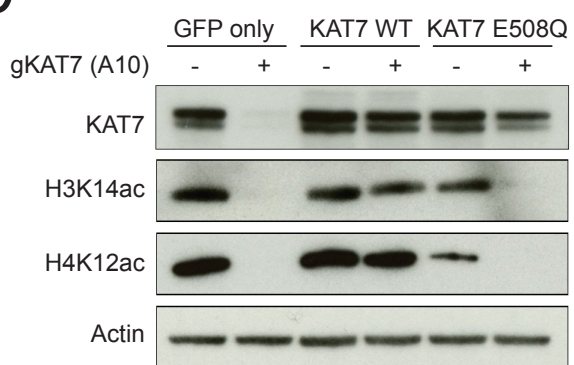

F
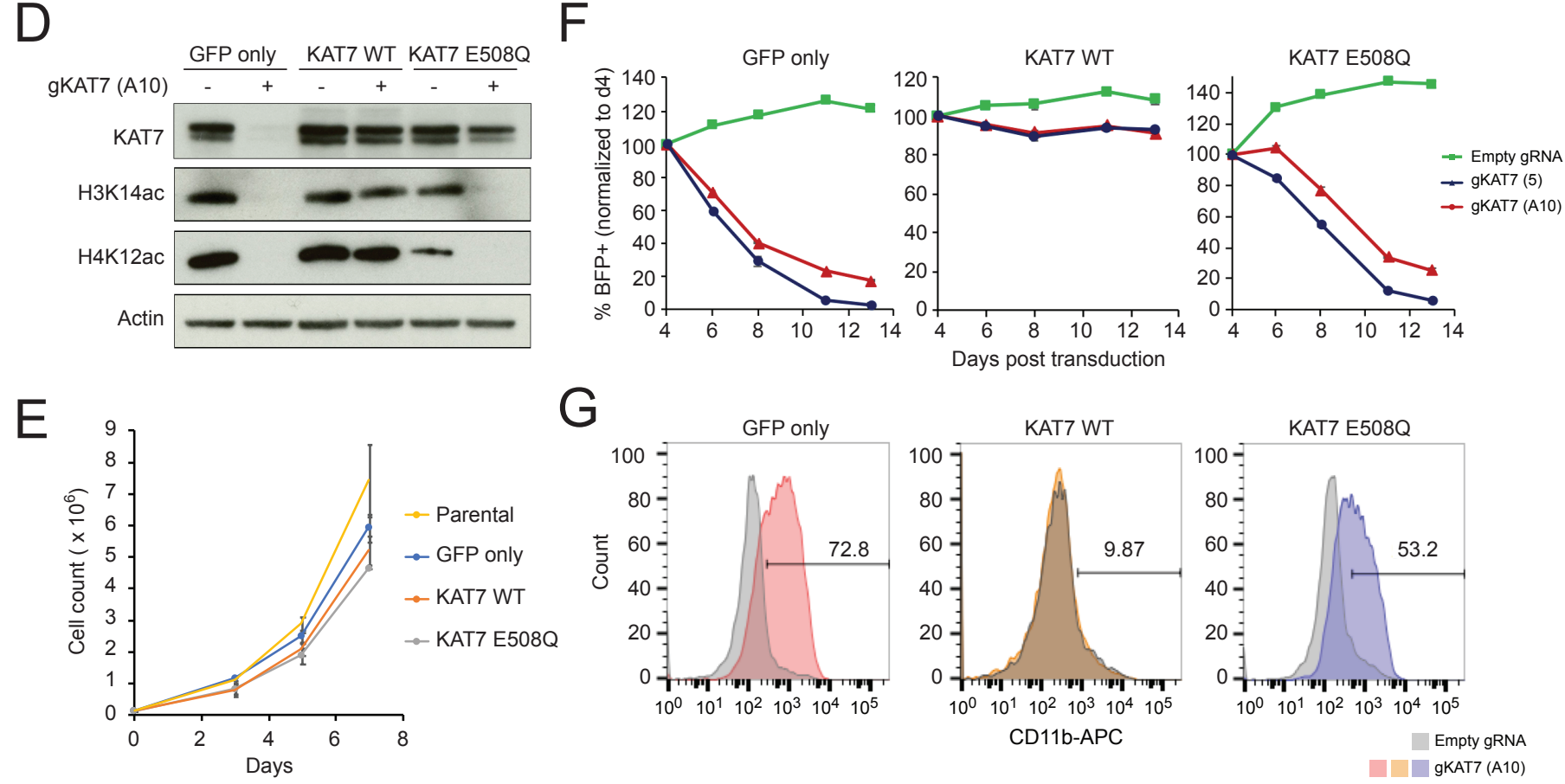
Figure 3

bioRxiv preprint doi: https://doi.org/10.1101/2020.04.25.054049; this version posted April 25, 2020. The copyright holder for this preprint (which was not certified by peer review) is the author/funder, who has granted bioRxiv a license to display the preprint in perpetuity. It is made
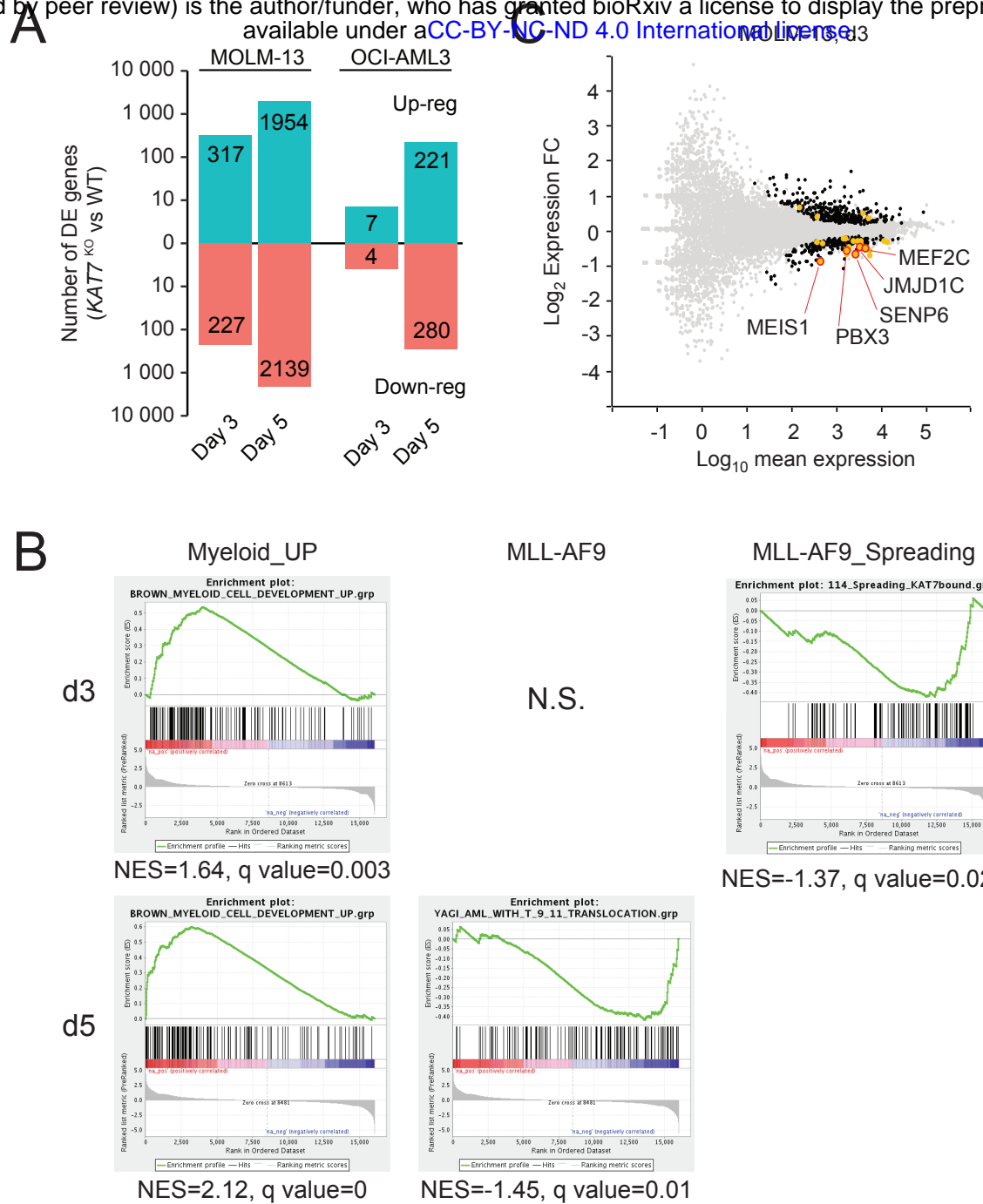

MLL-AF9

MLL-AF9_Spreading

N.S.

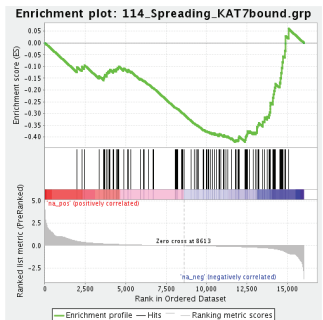

NES $=-1.37, \mathrm{q}$ value $=0.020$

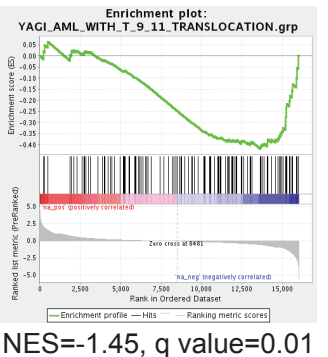


Figure 4

bioRxiv preprint doi: https://doi.org/10.1101/2020.04.25.054049; this version posted April 25, 2020. The copyright holder for this preprint (which was not certified by peer review) is the author/funder, who has granted bioRxiv a license to display the preprint in perpetuity. It is made
A
B avaliable under aCC-BY-NC-ND 4.0 International license.
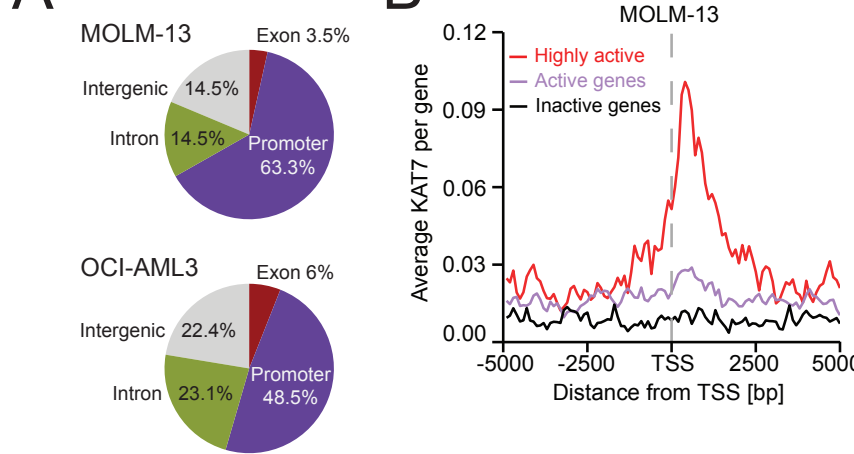
C ${ }_{\text {моцм-13 }} \mathrm{D}$

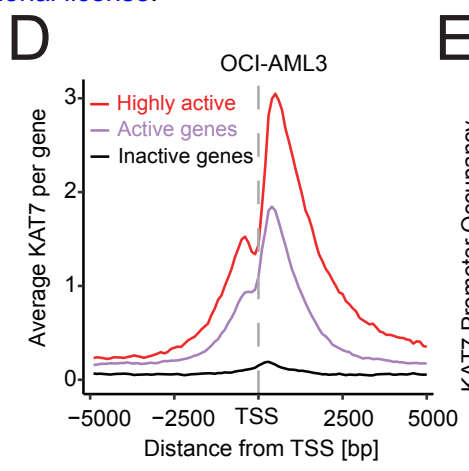
E осі-Aмเз
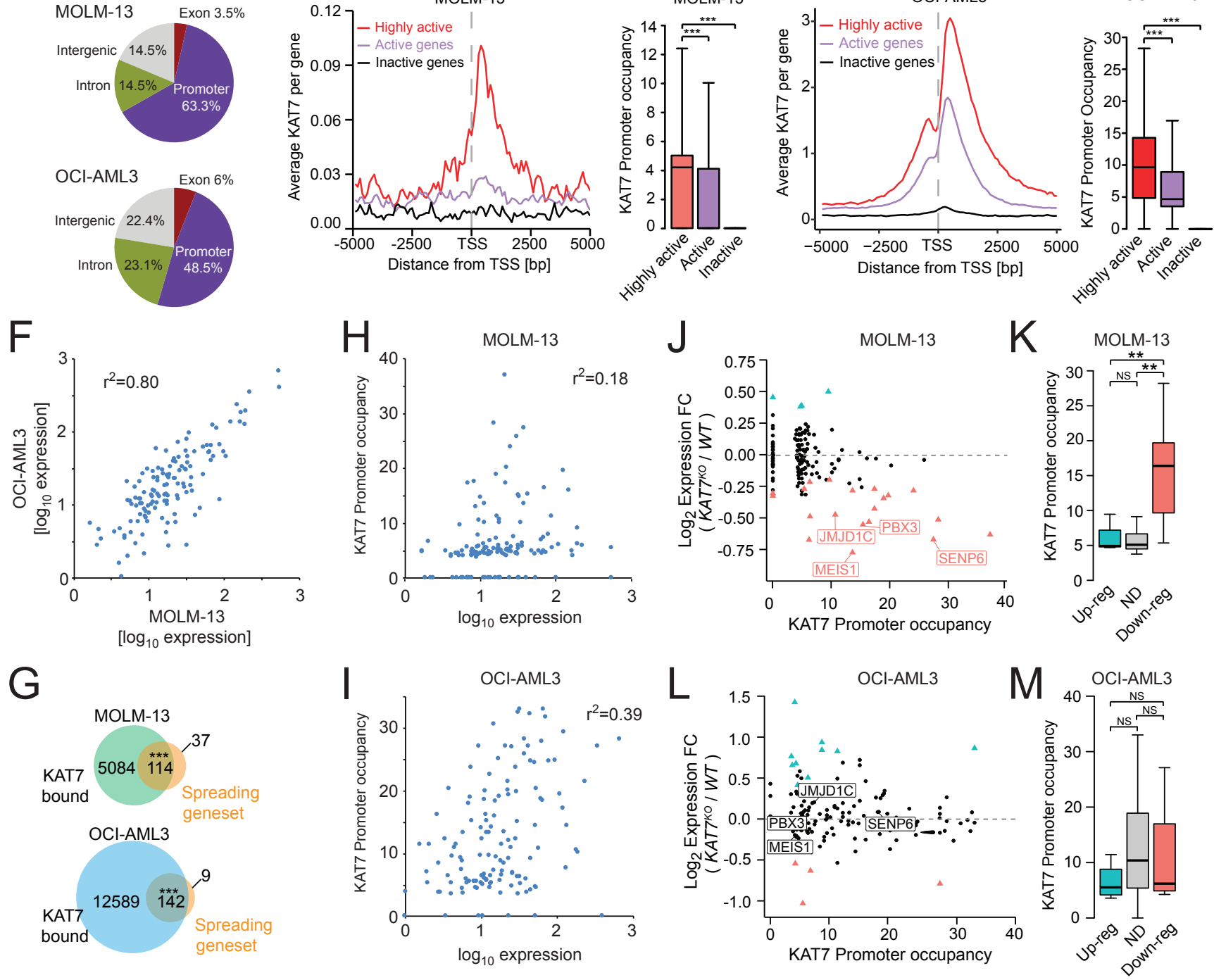
Figure 5

bioRxiv preprint doi: https://doi.org/10.1101/2020.04.25.054049; this version posted April 25, 2020. The copyright holder for this preprint (which was not certified by peer review) is the author/funder, who has granted bioRxiv a license to display the preprint in perpetuity. It is made

A available untgr aCC-BY-NC-ND 4.0 International license.
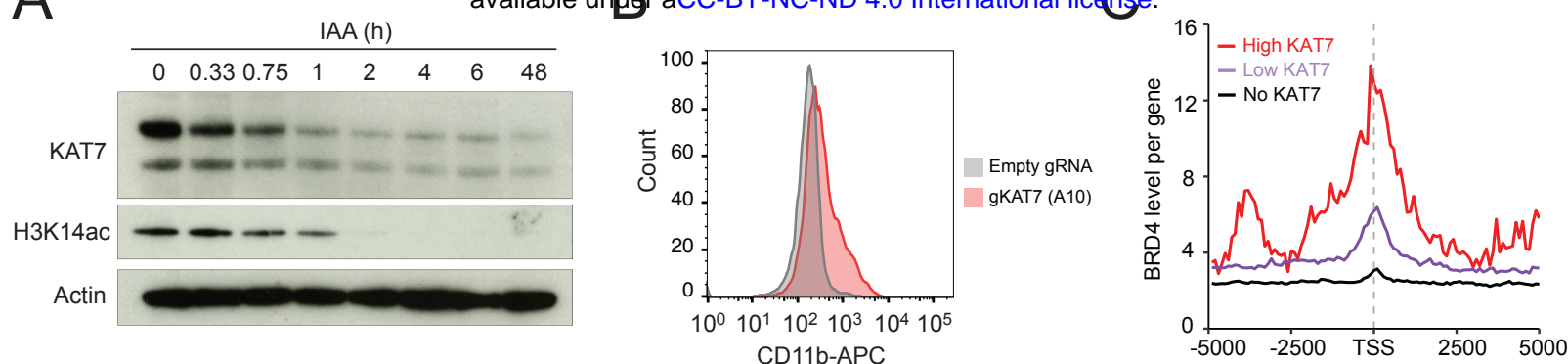

$\mathrm{D}$
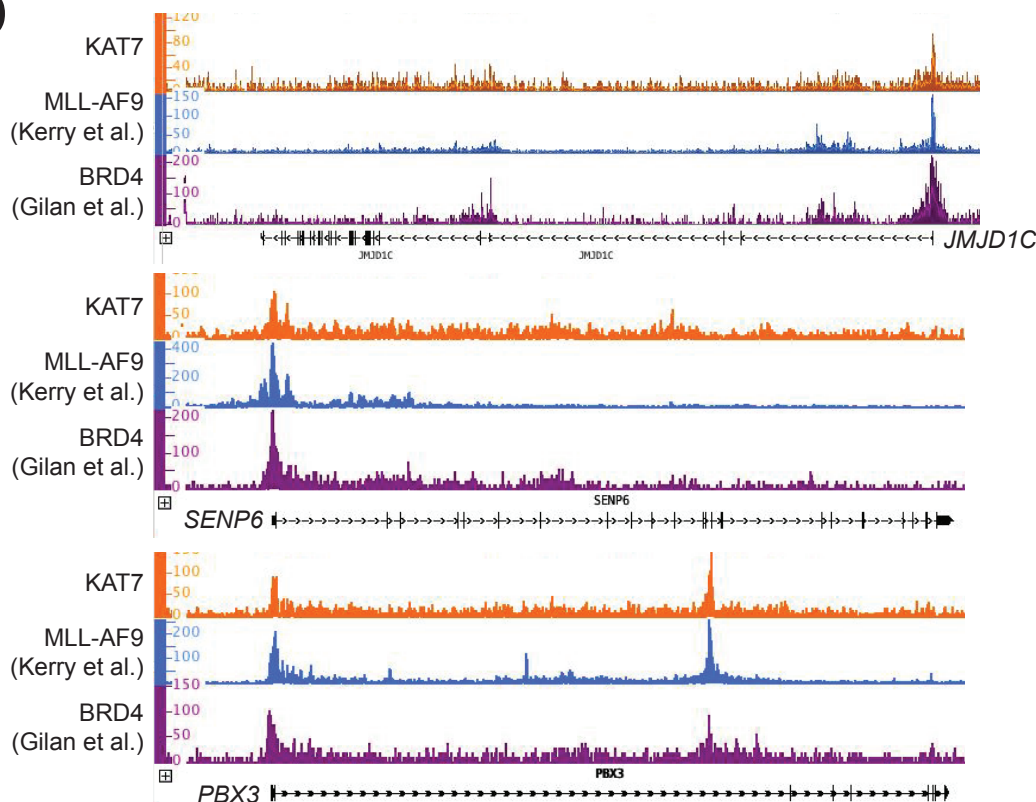

E

$\mathrm{F}$
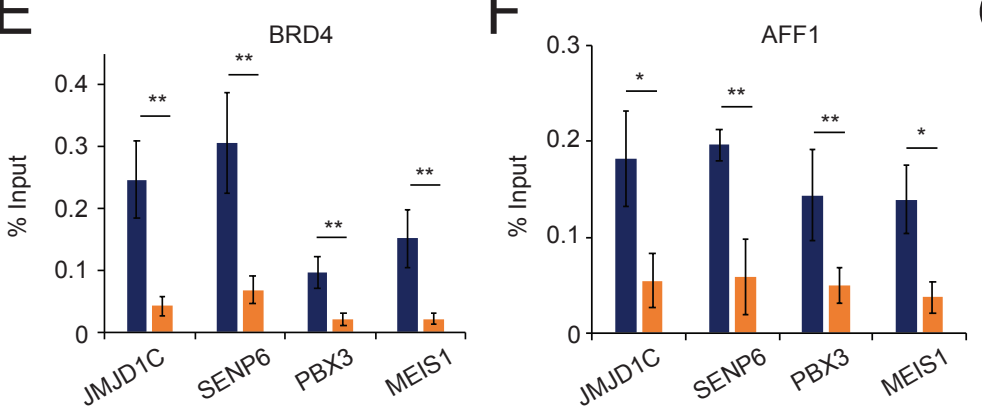

G Polll ps5

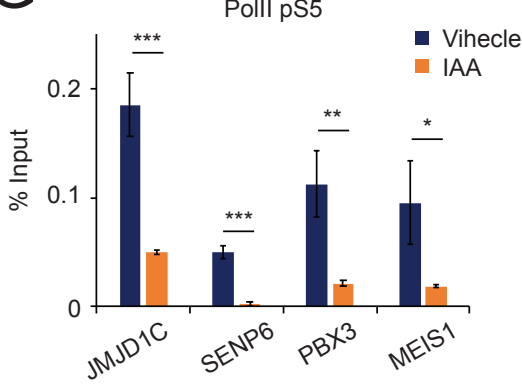

$\mathrm{H}$
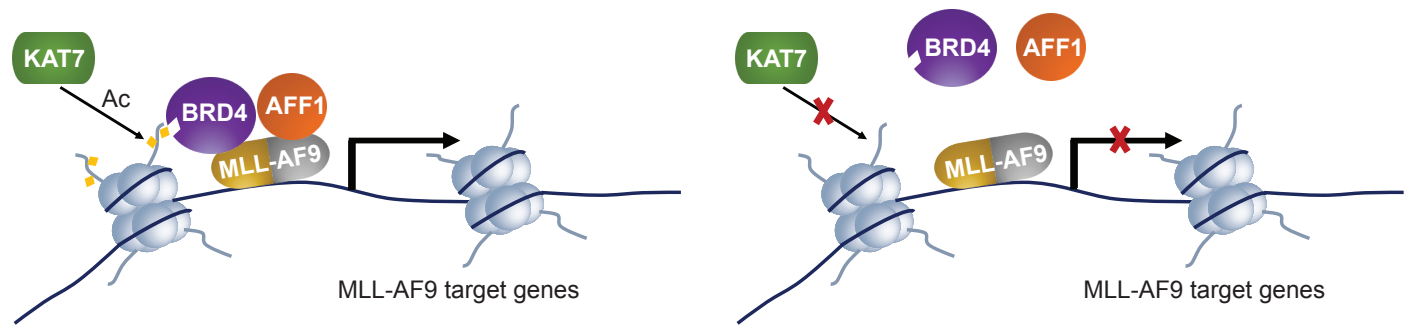